Geography as a science uses definite analytical methods to study the extremely complex object as defined so far. We distinguish a) the different systems of approach, i. e. the anorganic, organic and cultural geography and b) the formal and functional directions of approach. In both cases the landscape has to be viewed genetically. The combination of the different approaches leads towards an exact understanding of the whole of the landscape.

\title{
KLIMATOLOGIE IM LICHTE DER NEUEREN LEHRBÜCHER
}

\author{
Paul KaufmanN
}

Die Entwicklung der Klimatologie erhielt seit Jahrzehnten Impulse aus drei Richtungen. Sie befindet sich derzeit in einer Wandlung, welche noch keineswegs abgeschlossen ist, es aber doch schon ratsam erscheinen läßt, die Lehrbücher zu revidieren. Dies ist seit dem immer noch nicht vollendeten grundlegenden Handbuch der Klimatologie von W. KöPPEN und R. Geıger (Berlin $1932 \mathrm{ff}$.) schon mehrfach geschehen, worauf im folgenden an Beispielen hingewiesen werden soll. Zunächst hat die angewandte Klimatologie ihren Bereich ungeahnt erweitert. Mit dem Landbau haben manche andere Wirtschaftszweige - so etwa die Elektrizitätswirtschaft, Organisationen des Verkehrs usw. - Fragen der Praxis gestellt, welche zu beantworten die Klimatologie sich erst anschicken mußte. Die Landesplanung ist zusehends mehr auf die Mitarbeit praktisch ausgerichteter Klimatologen angewiesen ${ }^{1}$. Eindrücklich zeigt sich dies beim bisher größten Unternehmen dieser Art, im Tennessee-Valley und vielleicht noch stärker bei dem Großprojekt der Landschaftsgestaltung zwischen Kirgisensteppe und Tundra. Den Haupterfolg erhofft man sich dort gerade aus dem Eingriff in das klimatische Gefüge.

Die Klimatologie ist des weitern von der Meteorologie her um viele Forschungsergebnisse bereichert worden. Neben der Erschließung und Bereinigung von Beobachtungsreihen für weitere Klimaelemente ist das Vordringen in bisher unbekannte Gebiete der Arktis und der Ozeane zu nennen. Die größte Bedeutung gerade für die Klimatologie haben indessen die messende Erfassung der Atmosphäre in der Vertikalen und die neueren meteorologischen Erkenntnisse über die Strömungsverhältnisse der Atmosphäre. Viele Ergebnisse sind sowohl meteorologischer wie klimatologischer Art. Das zeigt sich besonders an der Entwicklung des Bildes von der allgemeinen Zirkulation.

Einen Ansto $\beta$ von innen möchte man sodann jene Besinnung auf das Ziel der Klimatologie nennen, welche von Bergeron und Hesselberg eingeleitet wurde. Ursprünglich galt es, den Aspekt, welchen das Klima dem Synoptiker bietet, zur Geltung zu bringen (BERGERON), anderseits, auf die Ursächlichkeit einzugehen (HesselberG). Weitgehend nimmt sich heute die Meteorologie der letzteren Aufgabe an. Das Hauptanliegen der Klimatologie betrifft denn auch heute weniger Einzelfragen als den Weg zur gesamthaften Erfassung und Darstellung des Klimas: die klimatologische Synthese.

\section{Die neueren Hauptiverke}

Zunächst seien einige neuere Unterrichtswerke vorgestellt:

Trewartha, G.T.: An Introduction to Climate. (McGraw-Hill, New York, London 1954, 406 Seiten). Das Buch ist für die ersten Hochschulsemester bestimmt. Der erste Teil behandelt die Klimafaktoren (Sonne und Breite; Land und Wasser; semipermanente Hoch- und Tiefdruckzellen; Wind und Luftmassen; Höhenlage; Gebirgszüge; Meeresströmungen; tropische Stürme) und die Klimaelemente (Temperatur; Niederschlag und Feuchtigkeit; Luftdruck; Wind). Nach den Entstehungsgebieten werden je maritime und kontinentale Polar- und Tropikluft unterschieden. Nach den thermischen Verhältnissen erweitert sich die Unterteilung: so ist z. B. mit cPKu eine kontinentale Polarluftmasse bezeichnet, welche vom Boden erwärmt wird (K) und auch in den höhern Schichten instabil geschichtet ist (u). Oder mTWs bedeutet eine maritime Tropikluft, welche vom Boden abgekühlt wird (W) und auch oben stabil geschichtet ist (s). Neben der Polarfront werden die Intertropik- und die Arktik-Front naturgemäß nur kurz erwähnt.

Die Klimaklassifikation folgt zur Hauptsache KÖPPEN (Tropische Regenklimate [A]; Trockenklimate [B], usw.). Neu fügt Trewartha dazu die Klimate hoch gelegener Gebiete (H). Die Einzelklimate werden allerdings teilweise anders abgegrenzt. So ist bei den gemäßigt warmen Regenklimaten (C) nicht ausschließlich nach dem Niederschlag $\left(C_{s}, C_{w}\right.$ und $C_{f}$ ) unterschieden worden, sondern nach $C_{s}, C_{a}$ und $C_{b}^{\prime}{ }^{\prime}\left(a=\right.$ wärmster Monat über $22^{\circ} \mathrm{C}, b=$ wärmster Monat unter $\left.22^{\circ} \mathrm{C}\right)$. KÖPPEN's und THORNThwAITE's Klassifikation werden daneben ausführlich behandelt.

Der kürzere zweite Teil beschreibt die Klimatypen (nicht nach Erdteilen), wobei jeweils eigene Abschnitte über Vorkommen, spezielle Geomorphologie, hydrographische Verhältnisse, Bodenarten und Vegetation beigefügt sind. Die Illustration ist reichhaltig. Der Temperaturgang wird jedesmal auf vier Arten (nach Jefferson, Hartshorne, Troll und KöPpen) gezeigt. Trewartha setzt sich anch mit Fragen der Didaktik und mit den neuen Bestrebungen in der Klimatologie auseinander. $\mathrm{Er}$ ist aber eher für das Hergebrachte, weil er findet, den Anwendungsgebieten sei damit besser gedient als mit der neuerlichen Hervorhebung des genetischen Gesichtspunktes.

${ }^{1}$.Vgl. Schirmer, H. : Die Verwendung klimatologischer Karten in der Landesplanung. Berichte des Deutschen Wetterdienstes in der US-Zone Nr. 38 (Weickmann-Heft) Bad Kissingen 1952, 139-143. 
Alıssow, B. P.: Die Klimate der Erde (ohne das Gebiet der UdSSR). (Berlin 1954, 283 Seiten.) Es ist die Übersetzung eines 1950 in Moskau erschienenen Buches; seine regionale (noch nicht übersetzte) Ergänzung ist: BoRIssow, A. A.: Klimaty SSSR (Die Klimate der UdSSR), Moskau 1949, das dieselbe Methode hat. - Die Kenntnis der meteorologischen und klimatologischen Grundlagen voraussetzend, macht sich der Verfasser zur Aufgabe, "die vorhandenen Angaben über die zur Bildung der Klimate führenden Prozesse in einem gesetzmäßigen System zusammenzufassen und die Möglichkeit einer Vorauserkennung der Klimabedingungen zu zeigen, indem man von der geographischen Breite des Ortes ausgeht, von der atmosphärischen Zirkulation und von den Besonderheiten der Erdoberfläche». Das Buch weist didaktische Vorzüge auf, hat aber eine schlechte und z. T. veraltete Ausstattung. Zur klimatischen Raumgliederung hält Ausssow zunächst folgende Luftmassen-Herdgebiete auseinander : die Zone der äquatorialen Luft, die Zone der tropischen Luft, die Zone der Luftmassen gemäßigter Breiten (= polare Luftmassen) und die Gebiete der arkischen Luft. Diese Unterteilung erweitert er um drei Übergangszonen, welche der jahreszeitlichen Schwankung der Fronten und der Verlagerung der Hauptluftmassen entsprechen : die Zone des äquatorialen Monsuns, die subtropische Zone und die subarktische Zone. Die Raumgliederung folgt also der lokalen Luftmassenausbildung und der Lage der Hauptfronten. Verglichen mit der KöpPEN'schen ist sie zwar stärker zonal ausgerichtet. Die regionale Behandlung ist nach Erdteilen angelegt. Eingangs findet man jeweils eine Skizze der allgemeinen Zirkulation im betreffenden Gebiet (Luftmassenausbildung, Strömungsverhältnisse, jahreszeitliches Verhalten). Daraus wird dann das regionale Klima in den Grundzügen hergeleitet. Freilich muß sehr vieles noch rein beschreibend ergänzt werden. Die Erklärung bleibt oft wenig befriedigend. Eine detailliertere Durchführung der genetischen Behandlung würde besondere systematisch-statistische Untersuchungen erfordern. Diese fehlen hier, sollen aber in dem entsprechenden Buch über die UdSSR durchgeführt sein.

Gentilli, J.: A geography of climate (The University of Western Australia, Crawley 1952, 107 Seiten) ist ein handliches Repetitorium. Einige originelle bildliche Darstellungen können im Unterricht von Nutzen sein. KöPPENs und THORNTHWaITES Klimaklassifikationen sind auf 11 Seiten znsammengefaßt. Eine regionale Beschreibung fehlt. Doch bezieht der Verfasser auch dynamischkomplexe Erscheinungen in den Kreis seiner Betrachtungen, die besonders aus ihrer australasiatischen «Sicht» interessant sind.

Miller, A. A.: Climatology (Methuen, London 1949, 335 Seiten) gibt einen gleichfalls relativ knappen Überblick über die Regionalklimate. Die letzten Ergänzungen erfolgten übrigens 1944. Der Geograph findet wertvolle Einzelheiten z. B. bioklimatologischer Art. Die Darstellung trägt jedoch den neuesten Entwicklungen kaum mehr Rechnung.

Børman, E. W.: Klimaat-Klimaattypen-Klimaatgebieden-Bodenproductie-Bevolkning (2. Auflage, Noorduijn's Wetenschappelijke Reeks Nr. 25, Gorinchen 1949, 258 Seiten) bedient sich bei seiner regionalen Klimabeschreibung der Köppenschen Klimaklassifikation. Unter den auf die Anwendung gerichteten Abschnitten ist besonders derjenige über den Bodenertrag hervorzuheben.

Kendrew, W. C.: Climates (4. Aufl., University Press, Oxford 1949, 340 Seiten). Dieses viel erwähnte Lehrbuch gibt vor allem eine Darstellung der Wetter- und Klimaelemente und ihrer Verbreitung. Von Klimaten werden nur einige Typen (Sudan, Mittelmeer usw.) behandelt.

Haurwitz, B. and Austin, J. M.: Climatology (McClaw-Hill, New York und London 1944, 410 Seiten) dürfte eines der ersten klimatologischen Werke sein, das die Forderungen BERGERONs nach Berücksichtung der synoptischen Meteorologie zu verwirklichen trachtete. Es behandelt demnach auch die Luftmassen und Fronten und trägt sowohl der tages- als auch der jahreszeitlichen Dynamik Rechnung. Dabei sind sich die Verfasser bewußt, daß in dieser Sicht mehr meteorologische als geographische Betrachtungsweise zum Ausdruck kommt.

Strahler, A. N.: Physical Geography (John Wiley and Sons, New York, London 1951, 451 Seiten) bietet eine relativ eingehende moderne Klimadarstellung. Sie nimmt etwa den dritten Teil seines Buches ein und basiert vornehmlich auf der Luftmassendifferenzierung. STRAHLER unterscheidet demzufolge drei Hauptklimagruppen : tropisch-äquatorial, tropisch-polar und arktisch-polar bestimmte, denen er 13 Großklimate zuordnet. Ihre Darstellung erfolgt mittelst origineller Illustrationen.

\section{Die Grundtendenzen}

Es ist wohl angebracht, neue Lehrbücher im Zusammenhang mit den Tendenzen des Fachgebietes zu betrachten. Diese hat H. FLoHN (Witterung und Klima in Mitteleuropa. Zürich 1954) aufgezeigt. Außerdem erfolgte eine Orientierung durch M. SchüEpp ${ }^{2}$. Darnach läßt sich die derzeitige Entwicklung wie folgt zusammenfassen: Seit W. KöPPEN's letzter neubearbeiteter Auflage (1931) des aGrundrisses der Klimakunde" hat sich die sogenannte klassische Klimatologie konsolidiert. Da KöPPEN's Klimatologie in fast alle Lehrbücher eingegangen ist, gilt dies allgemein. Seither haben sich die Einwände und Verbesserungsvorschläge - aber auch die Vorarbeiten derart gemehrt, daß bereits von einer "modernen Klimatologie» (FLOHN) die Rede ist. Immerhin muß man der alten Klimatologie einiges zugutehalten: Einmal kann man ihrer auch in Zukunft nicht entraten; man will sie deshalb vor allem ergänzen, so auch hinsichtlich der Verwirklichung von Vorschlägen, die sich bereits in J.v. HANN's und KöPPEN's Schriften finden, welche heute erneut

${ }^{2}$ Der derzeitige Stand der Forschung in Meteorologie und Klimatologie. Geographica Helvetica, IX, 1954, 336-338. 
aufgegriffen werden, die mangels Beobachtungsmaterial aber nicht auszuführen waren. Wesentlich erscheint, daß an der Definition des Klimas, nämlich dem "mittleren Zustand und gewöhnlichen Verlauf der Witterung an einem gegebenen Orte» nicht gerüttelt wird. Das Programm verlegte nur seinen Schwerpunkt vom ersten auf den zweiten Teil der Definition.

Die klassische Klimatologie wurde vor allem als Mittel-wertklimatologie abgeschätzt, da Mittelwerte dem Verhalten der klimatischen Elemente nicht angemessen seien. Als ergänzende Angaben zum Mittelwert finden sich jetzt in der Fachliteratur vielfach solche über den Streubereich, die Periodizität und die Singularitäten der Zeitfolgen, ferner Abhängigkeitsmasse für das Verhalten verschiedener Elemente. Die bisher behandelten Klimaelemente machen nicht das aus, was wir am Wetter empfinden. Schon früh zeigte sich, da $ß$ die Elemente nicht isoliert wirken. Dies regte die sog. Komplex-Klimatologie an, welche u. a. von E. E. FEDOROW seit den Dreißigerjahren gefördert wird. (Vergl. PelzL, E.: Komplex-Klimatologie als witterungsklimatologische Untersuchungsmethode. Annalen der Meteorologie Bd. 7 [1955/56] S. 35-38.) In der Mittelwertklimatologie wird eine Beständigkeit vorgetäuscht, ein Verharren, welches nicht existiert. Man stellt nun jener Statik des Klimas die dynamische Klimatologie gegenüber, dem Zustand den Vorgang. Diese Entwicklung ging von der synoptischen Meteorologie aus. Die örtliche Abfolge und die Unterschiede des Wetters von Ort zu Ort sind nicht willkürlich. Sie erklären sich aus den verschiedenen Luftmassen und dem Ablauf der Strömung, sowie erdfesten Einflüssen. Luftmassen-Klassifikation und Fronten-Schema gehen hier von der Synoptik in die Klimatologie über. Die Ergänzung zur klassischen Klimatologie besteht in der sog. Witterungsklimatologie, welche (nach FLoHs, a. o. S. 18) "typische Einzelwetterlagen auswählt und versucht, diese kurz, aber eindeutig zu kennzeichnen. Sie beschreibt deren Verhalten in den einzelnen Gebieten und deren örtliche Abwandlungen. Sie kennzeichnet die Witterungen als eine in sich zusammenhängende Folge von Einzelabschnitten des Wetters...». Man kann den hier betonten Aspekt des Klimas kurz als gewöhnliche Folge typischer atmosphärischer Vorgänge fassen. Vielfach gerügt wurde ferner das Fehlen der Synthese. Es zeigte sich dabei, daß man sich nicht einig ist, was damit gemeint sei. Wir setzen Synthese gleich Gesamtschau. Sicher war diese mangelhaft, aber sie fehlte nicht, sie liegt in den Klimatypen. In deren Definition ist das zusammengefaßt, was man seinerzeit als ausschlaggebend betrachtete. Der Weg zur Synthese wird auch weiterhin derselbe sein. Teilweise wird unter Synthese auch das Aufzeigen der ursächlichen Zusammenhänge verstanden. Die klassische Klimatologie war vornehmlich beschreibend, da die Grundlagen zur Erklärung noch ausstanden. Manchen Beitrag hierzu hat inzwischen die Meteorologie geliefert. Vor allem weiß man jetzt über den bedeutendsten Klimafaktor, die allgemeine Zirkulation wesentlich besser Bescheid. Aber eine theoretische Klimatologie gibt es dennoch nicht, wenn auch Anfänge vorhanden sind, so etwa bei Milankovitch (Strahlungsklima). Diese Punkte werden von den meisten Autoren irgendwie erwähnt. Sie setzen dann auch zur Neugestaltung an: Die meteorologische Einführung ist erweitert, insbesondere bei den Abschnitten über Luftmassen, Dynamik der Zyklonen und die wetterhaften Vorgänge. Eine Reihe neuerschienener Werke der Meteorologie standen hierbei zur Verfügung. Damit wurden gleich zwei Ziele erreicht: Der meteorologische Teil leitet den Übergang von der statischen zur dynamischen Klimatologie ein, indem er die Einzelvorgänge und die Gesamtabläufe behandelt. Zugleich ermöglicht er vielfach den Übergang von der bloßen Beschreibung zur Erklärung. Bedeutend weniger wurde im Bezug auf die andern Punkte getan. Bezeichnend ist somit vor allem die Ausrichtung nach der heutigen Meteorologie.

Ausblick

Die Klimatologie hat eine eigenartige Stellung zwischen Meteorologie und Geographie: aus historischen und methodischen Gründen zählt sie noch als geographische Disziplin, doch sie lebt längst so gut wie ganz auf Kosten der Meteorologie. J. v. HANN betrachtete die Klimatologie als "den mehr praktischen und geographischen Teil der Meteorologie", und er setzte klimatologisch mit statistisch gleich, meteorologisch mit physikalisch. Doch hat inzwischen Besinnung und Wandlung eingesetzt. Das Zentralproblem der gegenwärtigen Klimatologie liegt unverkennbar in der Synthese und damit in gewissem Sinn auf der Klimasystematik ${ }^{3}$. TREWARTHA deutet das im Zusammenhang mit der Systematik an, wenn er u. a. sagt: "Thereby simplicity and order are introduced into what at first may have been a bewilderung multiplicity of individuals. Classification there by aids in establishing general truths from numerous individual instances." S. (224). Alsssow will "auf genetischer Grundlage..., den Gesamtkomplex der zu einem gegebenen Klima gehörigen meteorologischen Erscheinungen erfassen und auf diese Weise eine vollkommenere Charakteristik des Klimas geben...». Hier zeigen sich zwei Komponenten der Synthese: Gesamtschau und Erklärung. Sie sind komplementär, können sich gegenseitig nicht vertreten. Das erkennt man etwa in den Mängeln einseitig gehaltener Lehrbücher. Die Synthese ist auf die Systematik wie auch auf die Ursachenforschung angewiesen. An sich besteht zwar eine Alternative: Die Gesamtschau kann entweder mittels des Ordnens der Mannigfaltigkeit (Systematik) oder mittels des Zuordnens der

3 Uber deren Verfahren referieren: K. KNoch und A. Schultze: Methoden der Klimaklassifikation. Gotha 1952. Hierzu kritisch: H. LAUTENSACH: Methoden der Klimaklassifikation. Peterm. Geogr. Mitteil. 98, 1954, 198-199, mit anschließender Erwiderung durch KNoch und Schultze. Vgl. Geographica Helvetica, IX, 1954, 51-52. 
Beobachtungen nach kausalen Verbindungen erreicht werden. Beides - und insbesondere das letztere - ist aber nur in ganz beschränktem Maße durchführbar, wie anschließend gezeigt werden soll. Der erste Weg beginnt schon bei der Ordnung der Wetterelemente. E. E. FEDorow hat dazu nach der Kombination der Wetterelemente ein System (von $10^{8}$ Typen) entwickelt. Die Witterungsklimatologie wird die vorherrschenden Elementenkombinationen bei den typischen Wetterlagen herausarbeiten und dann die Frequenzen der Witterungen, sowie ihre Besonderheiten in der Zeitfolge feststellen. F. BAUR hat dazu einen Katalog der Großwetterlagen (deren Zahl von 15 inzwischen auf 28 gestiegen ist) gegeben. Bei dieser schrittweisen Klassierung wird die Synthese dem Gedächtnis überbunden: Es muß die Definitionen der Kombinationen beherrschen und die zugehörigen Frequenzen zur Verfügung haben. Die Intensität der Synthese ist natürlich durch die Leistungsfähigkeit des Gedächtnisses beschränkt, soweit es sich um selbständige Geistesarbeit handelt. Dazu wird dann noch die räumliche Übersicht gefordert: Die örtliche Differenzierung der Elementenkombination und der Frequenzen der Wetterlagen, welche durch die Ausmaße und die Kinematik der Luftmassen, ferner durch die Lokalfaktoren gegeben ist, muß anschaulich dargestellt werden können. Die Aufforderungen an das Gedächtnis und das Vorstellungsvermögen steigern sich hier gewaltig. Dennoch verspricht einzig die Bewältigung der Synthese aus der Systematik und Statistik einigen Erfolg. Der andere Weg liegt etwa in folgendem: Zugrunde liegt ein mehr oder weniger geschlossenes physikalisches System, in welchem eine Reihe von Grundgesetzen gelten. Darunter finden sich das Grundgleichungssystem der dynamischen Meteorologie, aber auch die Gesetze der Strahlung, der Diffusionsvorgänge, der Wolkenphysik usw. Die Gesamtheit der Gesetze enthält nun die Synthese in kompaktester Form, denn ihre Lösung stellt den raum-zeitlichen Wetterablauf schlechthin dar, und daraus läßt sich jede klimatologische Frage beantworten. Freilich ist zuzugeben, daß der Mensch mit dieser Strukturgesamtheit nichts anfangen kann, ohne einen überaus leistungsfähigen elektronischen Roboter zu Hilfe zu nehmen. Das gilt aber auch bei einer intensiveren Synthese auf dem Wege über die Systematik. Der letztere hat aber den Vorteil, daß dort keine prinzipiellen Schwierigkeiten vorliegen, während beim erstern schon die Gesamtheit der Grundgesetze nicht vollständig angegeben werden kann, somit die Komplexität des Gegenstandes nicht beherrscht wird. Eine andere Schwierigkeit hängt mit der Frage zusammen, wie weit das System überhaupt geschlossen sei. So sieht der deduktive Weg bei einiger Strenge aus. Der erste, auf Systematik und Statistik beruhende Weg ist empirischer (also im Grunde induktiver) Art. Beide reihen sich in die genetische Betrachtungsweise ein. Alssow versucht die behelfsmäßige, unvollständige und rein qualitative Durchführung der Deduktion. Eine eingehendere Darstellung des Klimas auf genetischer Grundlage wird jedoch empirische (systematisch-statistische) Methoden benützen müssen, welche die Witterungsklimatologie erst entwickelt. Erklären bleibt hier eine Kunst des Möglichen, und das geläufige Plausibelmachen verkennt nur zu oft die Situation, welche wir zuvor anzudeuten versuchten. Es fiele nicht schwer, die Parallelen bei der landschaftskundlichen Synthese aufzuzeigen.

Das Sachgebiet der Klimatologie fällt mit demjenigen der Meteorologie zusammen; die Probleme jedoch entstehen bei ihr aus einer ganzheitlichen, $d$. h. bereits auf die Synthese ausgerichteten Betrachtungsweise, welche vor allem der theoretischen Meteorologie fremd ist. Die Klimatologie steht offensichtlich unter dem Zwange zur Synthese, und dieser hält ihre Entwicklung zurück. Weiter ist durch die Problemlage die Wahl der Prinzipien bedingt: diese müssen der komplexen Natur des Gegenstandes entsprechen; es können nicht einfach physikalische Einzelgesetze übernommen werden. So weist denn die Klimatologie eigentlich nur Klassifikationsprinzipien auf. Da hängt dann der Erfolg wesentlich von der Wahl der Verfahren ab, und dafür ist das fortwährende Suchen nach Methoden bezeichnend. Die durch das Wetter als Ganzes gegebene Problemlage (es geht hier nicht um Ganzheit im Sinne der Autonomie, sondern um die Vollständigkeit in der Erfassung der Struktur der Erscheinung) grenzt die Klimatologie von der Meteorologie als Physik der Atmosphäre ab. Ohne dieses synthetische Moment wäre die Klimatologie lediglich regionale Meteorologie. Es ist noch ungewiß, wann und wie die neuere Entwicklung der Klimatologie maßgebend Einfluß auf den Unterricht gewinnen wird. Die empirische Methode müßte jedenfalls den Gedächtnisstoff weiterhin vermehren. Die Gesamtschau wird auch dann noch eine beträchtliche Geistesarbeit erfordern.

Die Erklärung aus der allgemeinen Zirkulation setzt wohl auch mehr Kenntnisse über die letztere voraus, als bisher bereitgestellt wurden. Ein eingehenderes Studium dieser Art verlangt jedenfalls synoptische Erfahrung. Alsssow wendet sich mit den genetischen Gedankengängen in seinem Buche nur an die fortgeschrittenen Semester. Die Erklärung kann sich aber auf der Mittelschulstufe auf die Grundzüge des Klimas beschränken. Da scheint uns bei einer gewissen Änderung der Stoffauswahl der deduktive Weg im Bereich des Möglichen zu liegen. Entscheidend wird wohl sein, welcher Weg den rationelleren und zum Denken anregenderen Unterricht ergibt. Eine übersteigerte Systematik und Statistik wird sich weder bei klassischer noch bei moderner SchulKlimatologie einfügen. Dagegen könnten Alıssow's einleitende Kapitel über die Grundzüge der Klimate der einzelnen Erdteile brauchbare Hinweise geben.

Daß mit Trewartha die meisten Autoren der modernen Entwicklung noch zurückhaltend gegenüberstehen, ist verständlich. Einmal liegen noch kaum witterungsklimatologische Regionaldarstellungen vor. Dann fehlen die Unterrichtserfahrungen wohl noch gänzlich. So bleibt abzuwarten, wie rasch die Schulen die modernen Anschauungen in ihr Lehrprogramm einbauen. 